\title{
CONDICIÓN DE ARRECIFES DE CORAL SOMETIDOS A PRESIONES NATURALES RECIENTES: BAJOS DE SANCHO PARDO, CUBA
}

\section{CONDITION OF CORAL REEFS UNDER RECENT NATURAL PRESSURE: BAJOS DE SANCHO PARDO, CUBA}

\author{
Hansel Caballero ${ }^{1 *}$ y Pedro M. Alcolado ${ }^{2}$
}

\begin{abstract}
RESUMEN
Se evaluó la condición de la barrera de coral de los Bajos de Sancho Pardo (NW Cuba), área alejada de acciones antrópicas (excepto sobrepesca), pero que ha sufrido un período de frecuencia de huracanes, brotes de enfermedades y eventos de blanqueamiento de corales. En agosto del 2010 se aplicó la metodología de muestreo AGRRA en comunidades de corales de tres estaciones en las crestas, tres en las zonas traseras y 15 en el frente de arrecifes. Se identificaron 32 especies de corales, considerando solo colonias mayores de $10 \mathrm{~cm}$ de diámetro máximo. Los indicadores biológicos analizados sugieren cierta heterogeneidad en las condiciones medioambientales y diferentes grados de resiliencia de los corales ante los disturbios naturales. El cubrimiento vivo medio de coral fue de $23 \%$ en las crestas, $27 \%$ en las zonas traseras y $9 \%$ en los arrecifes frontales. La densidad media lineal de corales fue de 8 colonias $/ 10 \mathrm{~m}$ en las crestas, 11 colonias $/ 10 \mathrm{~m}$ en las zonas traseras y 5 colonias $/ 10 \mathrm{~m}$ en los arrecifes frontales. Se observó mortalidad reciente por enfermedades activas. La mortalidad antigua fue mayor en las zonas traseras y en las crestas, a causa aparentemente de la mayor influencia de los huracanes por la menor profundidad. Los arrecifes estuvieron perturbados por cubrimiento importante de algas carnosas supuestamente por un insuficiente nivel de herbivorismo.
\end{abstract}

Palabras claves: AGRRA, arrecifes de coral, huracanes, disturbios naturales, Cuba.

\begin{abstract}
The condition of the barrier reef in Bajos de Sancho Pardo (NW Cuba) was assessed. This area is located far from anthropogenic disturbances (except overfishing) and has suffered a period of frequent hurricanes, coral disease outbreaks, and coral bleaching events. In August 2010, the AGRRA sampling methodology was applied to 21 coral communities; three crest stations, three rear zones, and 15 fore reef stations. Thirty-two coral species were identified, considering only colonies larger than $10 \mathrm{~cm}$ maximum diameter. The biological indicators analyzed suggest some degree of heterogeneity of environmental conditions and different levels of coral resiliency to natural disturbances. Average live coral cover was $23 \%$ on reef crests, $27 \%$ on back reefs and $9 \%$ on fore reefs. Average coral linear density was 8 colonies $/ 10 \mathrm{~m}$ on reef crests, 11 colonies $/ 10 \mathrm{~m}$ on back reefs and 5 colonies $/ 10 \mathrm{~m}$ on fore reefs. Recent mortality was observed resulting from active diseases. Old mortality was higher in back reefs and reef crests, apparently due to the higher influence of hurricanes due to their shallow depths. Reefs were disturbed by a significant fleshy algae cover provably due to low levels of herbivory.
\end{abstract}

Keywords: AGRRA, coral reefs, hurricanes, natural disturbances, Cuba.

1 Acuario Nacional de Cuba, Ave. 1ra. y 60, Playa, CP 11300, Ciudad Habana, Cuba.

2 Instituto de Oceanología, Ave. 1ra., No. 18406, Rpto. Flores, Playa, La Habana, Cuba. *hanselc@acuarionacional.cu

Recibido 18-I-2011

Aceptado 30-V-2011

DOI: http://dx.doi.org/10.15359/revmar.3.4 


\section{INTRODUCCIÓN}

El cambio climático es reconocido en la actualidad como una de las mayores amenazas a los arrecifes de coral en el mundo (Hoegh-Guldberg et al. 2007; Wilkinson \& Souter, 2008; Obura \& Grimsditch, 2008). Su efecto se pone de manifiesto con el incremento de disturbios de carácter natural debidos al cambio climático o a la variabilidad del clima (aumento de la intensidad de huracanes, aumento de la temperatura y acidificación del agua, enfermedades y blanqueamiento de corales) que han contribuido, junto a disturbios antropogénicos (sobrepesca, sedimentación terrígena y contaminación), al deterioro de la condición de las comunidades arrecifales (Aronson \& Precht, 2001; Veron et al. 2009; Alcolado, 2010).

Contrariamente a décadas pasadas, los arrecifes alejados de fuentes de contaminación e influencia terrígena están sufriendo daños en su estructura, composición y porcentajes de cubrimiento vivo de coral. Este es el caso de los Bajos de Sancho Pardo, barrera de coral del extremo noroccidental de Cuba, alejada $50 \mathrm{~km}$ de la isla principal. Esta área experimentó el impacto de seis huracanes entre el 2000 y el 2005 e igualmente sus arrecifes no deben haber estado exentos de la influencia de la activa temporada de enfermedades y blanqueamientos de corales de finales del siglo XX que mencionan Richardson (1998), Wilkinson (2000) y Aronson y Precht (2001).

La información previa publicada sobre las comunidades de corales de Sancho Pardo se limita a estudios realizados en el 2006 (González-Díaz et al. 2010). En este trabajo se presentarán los resultados de una evaluación rápida de los arrecifes de Sancho Pardo, área poco estudiada y, a su vez, sometida a presiones naturales. Tiene como objetivo evaluar la condición actual de estos, para que sirva como línea base para futuros monitoreos y análisis sobre la resiliencia de los corales ante el incremento de disturbios naturales en esta área geográfica.

\section{MATERIALES Y MÉTODOS}

\section{Descripción de la zona de estudio}

La barrera de coral de los Colorados (clasificada como tal por Walton-Smith, 1948), a la que pertenecen los Bajos de Sancho Pardo (con más de $40 \mathrm{~km}$ de extensión), se localiza en la región noroccidental de Cuba, al norte del Golfo de Guanahacabibes, formando parte del extremo occidental del Archipiélago de Los Colorados (Fig. 1). Los Bajos de Sancho Pardo están separados de la costa insular por una plataforma marina somera y relativamente amplia (50 km en la parte más ancha), que llega a alcanzar $25 \mathrm{~m}$ de profundidad.

En la zona geográfica predominan los vientos alisios del noreste durante gran parte del año, cuyo efecto es menor hacia la porción oeste de los arrecifes que están semirresguardados por su posición más a sotavento en el borde de la plataforma. Durante el invierno influyen de forma más directa e intensa vientos del norte y del noreste. La lejanía de los arrecifes con tierra firme hace que las concentraciones de nutrientes sean bajas (Lluis-Riera, 1983) y limita considerablemente la influencia antropogénica. No hay evidencias de ningún tipo de contaminación (González-Sansón et al. 2009).

A lo largo de la zona arrecifal somera (1-3 m) se apreciaron amplias y continuas formaciones calcáreas conocidas como "bajos", sin embargo, el desarrollo de las crestas (con crecimiento de Acropora palmata Lamarck, 1816) sobre estos no fue igualmente continuo, sino a manera de "parches" de 

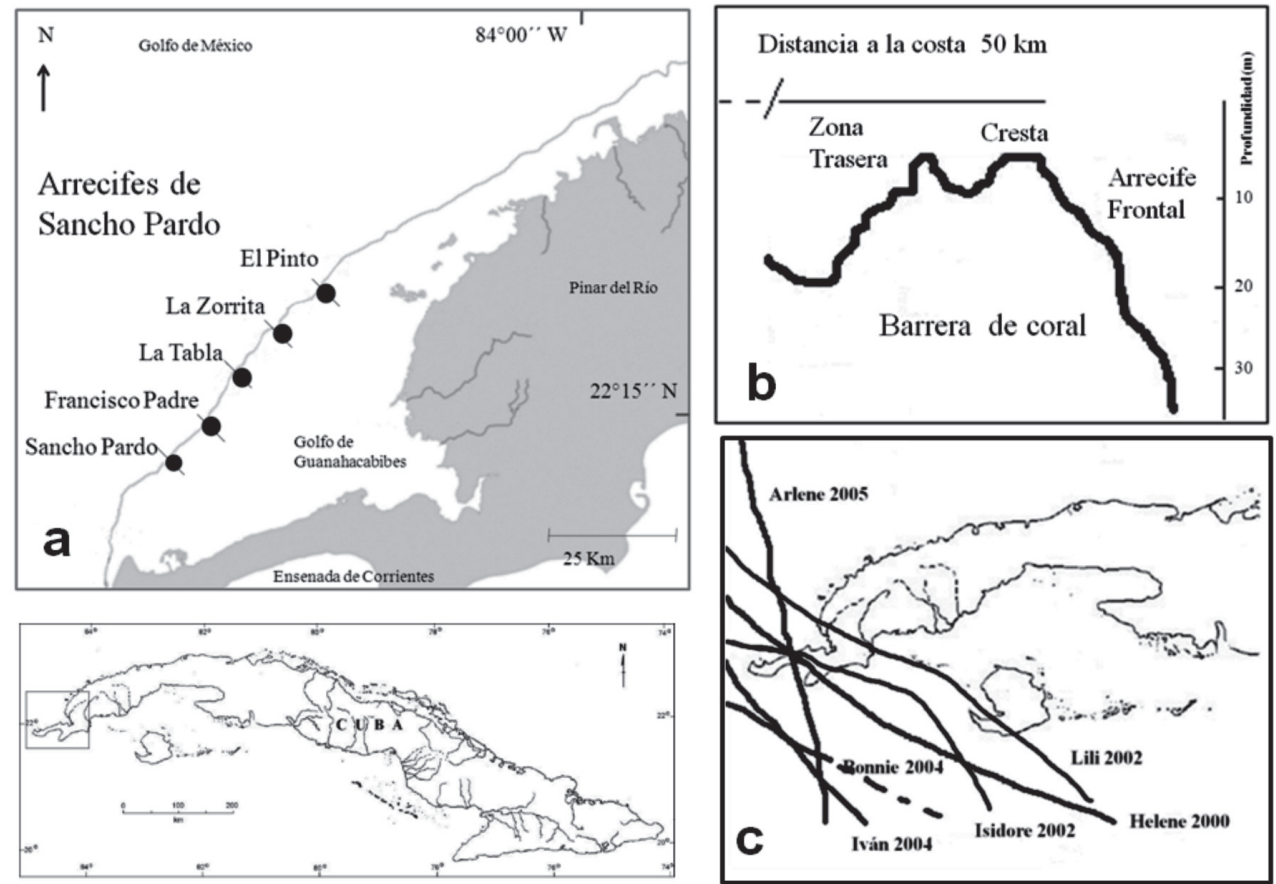

Fig. 1. Ubicación del área de investigación; a: ubicación geográfica de los sitios de muestreo; b: perfil del fondo del arrecife (modificado de Claro, 2006) y zonas ecológicas trabajadas; c: recorrido de huracanes por el área entre los años 2000 y 2005 según www.nhc.noaa.gov/

Fig. 1. Location of research area; a: Location of sampling sites; b: coral reef bottom profile (modified from Claro, 2006) and ecological zones researched; c: hurricane tracks on the area between 2000 and 2005 according to www.nhc.noaa.gov/

extensión variable. Como generalidad, a excepción de los sitios de crestas cuantificados en este trabajo, sobre los bajos se observó un predominio de corales incrustantes aislados y octocorales considerados resistentes al batimiento por Alcolado (1981). Las partes no colonizadas por corales en las crestas presentaron sustrato rocoso con predominio de algas costrosas y césped algal, así como una aceptable densidad $\left(2.5\right.$ ind.$\left./ \mathrm{m}^{2}\right)$ de erizos negros Diadema antillarum (Philippi, 1845).

Al oeste de la zona y localizadas continuamente al sur y al oeste de los bajos se observaron zonas traseras (4 $\mathrm{m}$ de profundidad) o resguardadas de "arrecifes de cabezos coralinos" muy densos. Se presentó un sustrato coralino alternado con parches de arena, con amplia fauna bentónica, donde predominaron Montastraea annularis (Ellis y Solander, 1876) y A. cervicornis (Lamarck, 1816). Las algas carnosas y calcáreas fueron abundantes.

Los arrecifes frontales a partir de los $8 \mathrm{~m}$ de profundidad aparecen como fondos bien definidos de camellones y canales (15 $\mathrm{m}$ de longitud, 2 a $5 \mathrm{~m}$ de anchura y $1 \mathrm{~m}$ de alto), que incrementan su complejidad y altura con la profundidad. En el sustrato del sitio más al oeste predominó un fondo coralino, a diferencia del resto, donde se destacó un pavimento rocoso, incluso a $20 \mathrm{~m}$ de profundidad. En los arrecifes frontales dominaron abundantemente las algas carnosas de los géneros Dictyota, Lobophora, Sargassum y Microdictyon. Los erizos negros estuvieron prácticamente ausentes. 


\section{Metodología y procesamiento de datos}

El muestreo se realizó en agosto del 2010. De oeste a este se establecieron cinco sitios o zonas de muestreo (Sancho Pardo, Francisco Padre, La Tabla, La Zorrita y El Pinto), separados aproximadamente $10 \mathrm{~km}$ entre sí, localizados sobre puntos de referencia ubicados por GPS (Fig. 1). Se trabajaron cuantitativamente las zonas ecológicas de arrecifes frontales (tres estaciones por cada sitio, ubicándose a $\operatorname{los} 10,15$ y $20 \mathrm{~m}$ de profundidad), las crestas (solo en Sancho Pardo, La Tabla y La Zorrita) y las zonas arrecifales traseras (solo en Sancho Pardo, Francisco Padre y La Tabla).

Tomando como base la metodología AGRRA (Kramer y Lang, 2003), se desplegaron transectos lineales (10 $\mathrm{m}$ de longitud) al azar dentro de tramos de aproximadamente $100 \mathrm{~m}$ de largo paralelos al borde de la plataforma. Para calcular el porcentaje de cobertura coralina, se determinó la proporción de cada transecto cubierto por tejido vivo de coral. Se cuantificaron las colonias interceptadas, se les determinó el diámetro máximo, se les examinó la presencia de enfermedades (se determinó, posteriormente, el porcentaje de colonias enfermas) y se estimaron los porcentajes de sus superficies que presentaron mortalidad reciente y mortalidad antigua. El diámetro se define como la dimensión máxima de la colonia vista desde arriba, la mortalidad antigua como cualquier área muerta en la que la estructura de los cálices ya no es visible y la mortalidad reciente como el esqueleto expuesto en el cual la estructura de los cálices aún es visible.

Para la identificación de los órdenes Scleractinia y Milleporina se siguieron los criterios de González-Ferrer (2009). Se incluyeron solamente las colonias con más de $10 \mathrm{~cm}$ de diámetro máximo. Se determinó la abundancia y composición por especies de corales según su porcentaje de cubrimiento del sustrato. Se determinó el porcentaje de abundancia atendiendo al cubrimiento de especies tolerantes al sedimento (ETS): $\mathrm{Si}$ derastrea siderea (Ellis y Solander, 1876), Porites astreoides (Lamarck, 1816), Montastraea cavernosa (Linnaeus, 1758), Diploria spp. y Meandrina meandrites (Linnaeus, 1758), Stephanocoenia intersepta (Esper, 1795), Millepora spp. y Porites spp., con base en los criterios de Acevedo et al. (1989), Rogers (1990) y Torres y Morelock (2002).

Se calcularon la media de los indicadores de cubrimiento vivo de coral, la densidad lineal, el diámetro máximo, las mortalidades antigua y reciente de las colonias. Para conocer la existencia de diferencias significativas entre sitios de arrecifes frontales se realizó un análisis de varianza bifactorial (ANOVA, sitio-profundidad), debido a la existencia de tres profundidades diferentes dentro de esta zona ecológica. Para la comparación entre sitios de crestas y zonas traseras, se aplicó un análisis de varianza unifactorial (ANOVA, sitios).

En todos los casos se comprobó la normalidad y homogeneidad de varianza de los datos (Zar, 1996). Se transformaron los datos mediante raíz cuadrada y cuarta cuando fue necesario. Para determinar las diferencias entre los pares de media se utilizó la prueba de Student-Newman-Keuls, con índice de significación de 0.05 . Todos los cálculos se realizaron con Statistica 6.0.

\section{RESULTADOS}

Las crestas mostraron un cubrimiento vivo de coral muy variable entre sitios (Fig. 2). En Francisco Padre fue prácticamente nulo. Ahí se observaron conglomerados de colonias $A$. palmata totalmente muertos, arrancados y en pie, sin muestras 

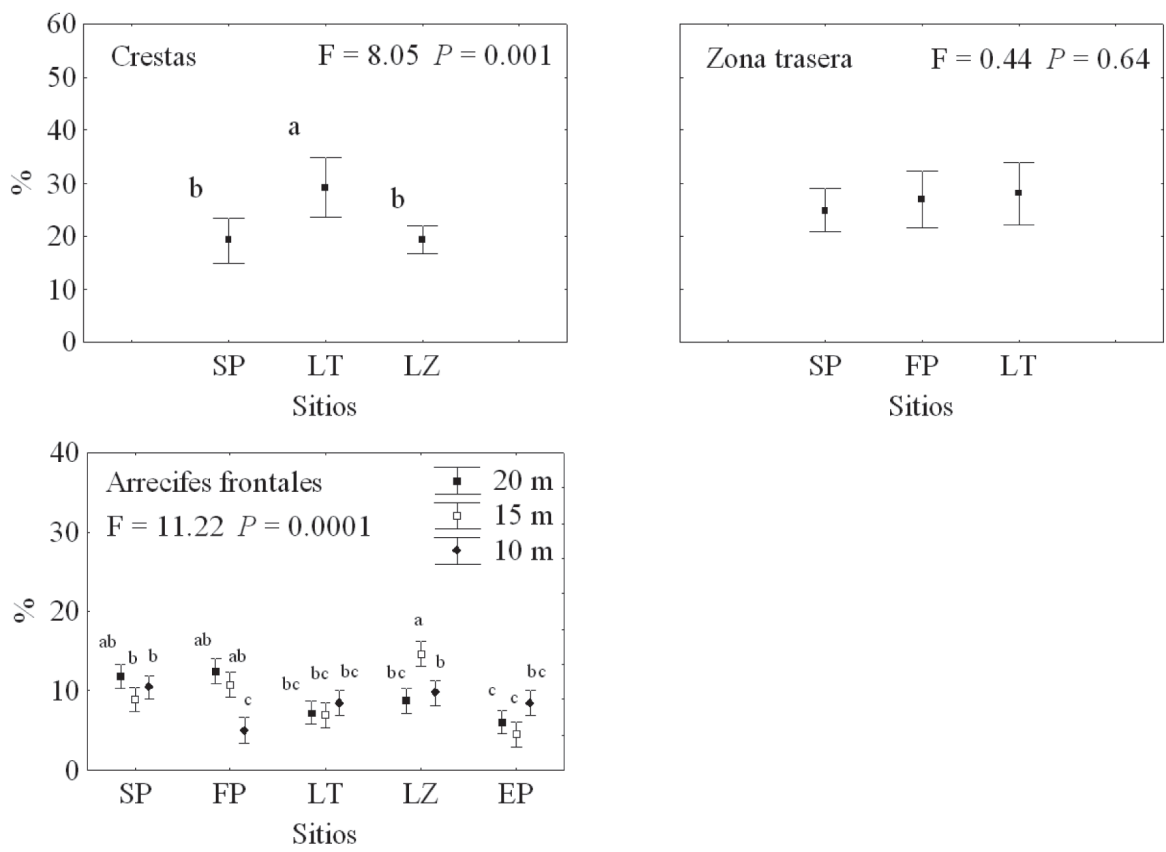

Fig. 2. Cubrimiento vivo de coral (media e intervalos de confianza) en las zonas ecológicas del arrecife. Las letras muestran las significaciones de las diferencias. $\mathrm{SP}=\mathrm{Sancho}$ Pardo, FP = Francisco Padre, $\mathrm{LT}=\mathrm{La}$ Tabla, $\mathrm{LZ}=$ La Zorrita y EP $=$ El Pinto

Fig. 2. Coral live cover (mean and confidence intervals) in the reef ecological zones. Letters indicate significance of differences. $\mathrm{SP}=$ Sancho Pardo, FP $=$ Francisco Padre, LT $=$ La Tabla, $\mathrm{LZ}=$ La Zorrita y EP $=$ El Pinto

de recubrimiento por tejido vivo. El resto de los corales fueron en su mayoría pequeñas colonias aisladas de $P$. astreoides y $S$. radians (Ellis y Solander, 1876).

En el sitio El Pinto tampoco se observó desarrollo de cresta arrecifal, sino "restingas" (restos de lo que fue una cresta) con aisladas colonias de corales incrustantes. En las restantes crestas, el cubrimiento medio varió de un $19 \%$ a un $30 \%$, siendo La Tabla significativamente superior.

Los sitios La Zorrita y El Pinto no presentaron zona arrecifal trasera, a continuación de los bajos solo se observaron pastos marinos. El cubrimiento vivo de coral en las zonas traseras de Sancho Pardo, Francisco Padre y La Tabla no mostró diferencias significativas entre sí y su valor promedio fue de $27 \%$ (Fig. 2). En los arrecifes frontales se encontró interacción sitio-profundidad en el análisis de varianza bifactorial. En general, el cubrimiento vivo de coral no sobrepasó el $15 \%$. Se destacaron por sus valores más altos los 10 y $20 \mathrm{~m}$ de Sancho Pardo, los 15 y 20 m de Francisco Padre y los $15 \mathrm{~m}$ de La Zorrita, con más de $10 \%$ de promedio. Fueron estadísticamente inferiores los cubrimientos de $10 \mathrm{~m}$ de Francisco Padre, y los 10 y 15 m de El Pinto (Fig. 2).

La densidad lineal media de corales en la cresta de Sancho Pardo fue significativamente menor que en el resto de los sitios, cuyos registros no superaron las 10 colonias/10 m. En las zonas traseras, las densidades sobrepasaron las 10 colonias $/ 10 \mathrm{~m}$, destacándose por un mayor valor Sancho 
Pardo. Los arrecifes frontales mostraron igualmente interacción sitio-profundidad. La Zorrita, a $15 \mathrm{~m}$, mostró la densidad más alta $(8$ colonias $/ 10 \mathrm{~m})$. El resto de los sitios varió con registros medios aproximados a 5 colonias/10 m (Fig. 3).

El diámetro máximo promedio de las colonias en las crestas varió de $40 \mathrm{~cm}$ en La Zorrita (significativamente menor) a 65 $\mathrm{cm}$ en La Tabla, mientras que en las zonas traseras varió de $45 \mathrm{~cm}$ en Sancho Pardo (diámetro estadísticamente menor) a 52 $\mathrm{cm}$ en las restantes. Los arrecifes frontales mostraron interacción sitio-profundidad. Los registros estadísticamente más altos los presentaron los sitios de Sancho Pardo y Francisco Padre, con promedios por encima de $30 \mathrm{~cm}$ (Fig. 4).
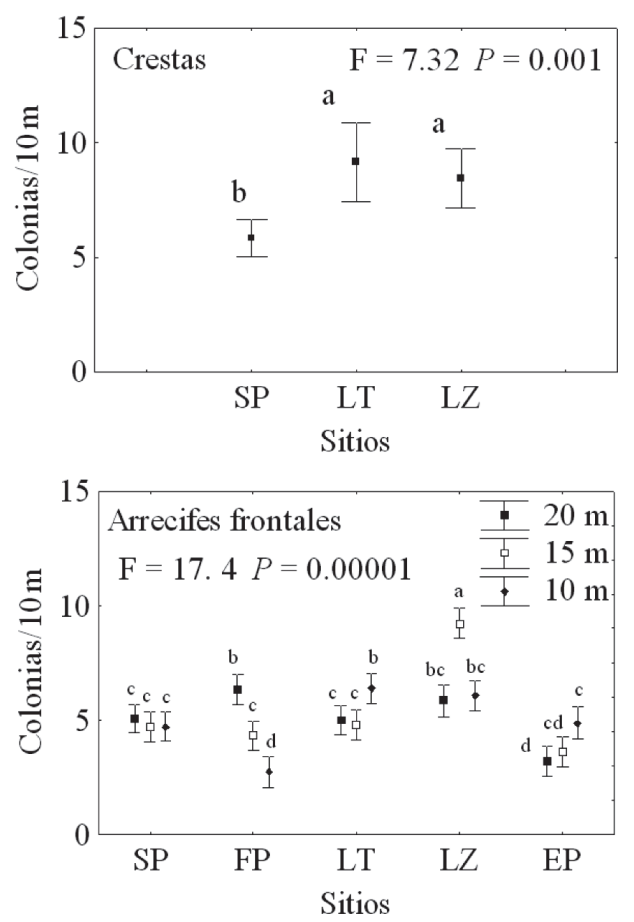

No se encontraron diferencias significativas entre los promedios de mortalidad antigua, tanto en crestas como en zonas traseras $(20 \%$ y $29 \%$, respectivamente). Los arrecifes frontales mostraron interacción sitio-profundidad. La estación de 20 $\mathrm{m}$ de La Tabla presentó mayor mortalidad con un promedio de 19\% (Fig. 5).

Se encontraron enfermedades activas. La "plaga blanca" llegó a infestar hasta un $9 \%$ de colonias en los arrecifes frontales. Las "manchas oscuras" se presentaron en menor frecuencia. Durante los muestreos prácticamente no se vio blanqueamiento (Cuadro 1).

Se identificaron 30 especies de corales pétreos y 2 especies de hidroides calcificados, de un total de 2072 colonias

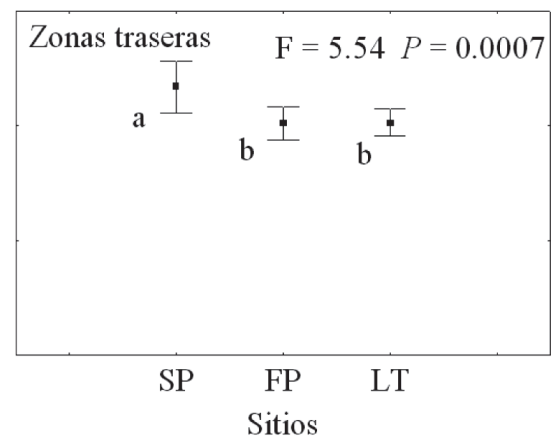

Fig. 3. Densidad de corales (media e intervalos de confianza) de las zonas ecológicas del arrecife. Las letras diferentes muestran la significación de las diferencias. Ver siglas de los sitios en la Fig. 2 Fig. 3. Coral density (mean and confidence intervals) of the reef ecological zones. Letters indicate significance of differences. Site abbreviations codes as in Fig. 2 

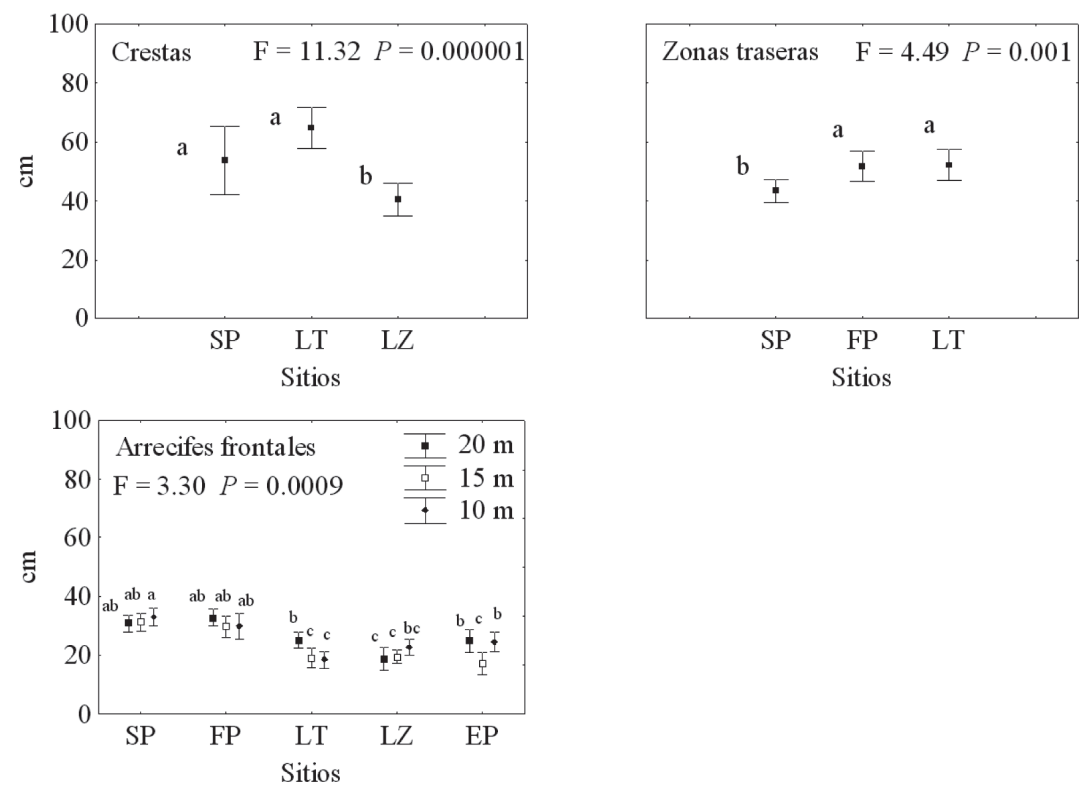

Fig. 4. Diámetro máximo de las colonias de coral (media e intervalos de confianza) de las zonas ecológicas del arrecife. Las letras muestran la significación de las diferencias. Ver siglas de los sitios en la Fig. 2 Fig. 4. Maximum diameter of coral colonies (mean and confidence intervals) of the reef ecological zones. Different letters indicate significance of differences. Site codes as in Fig. 2
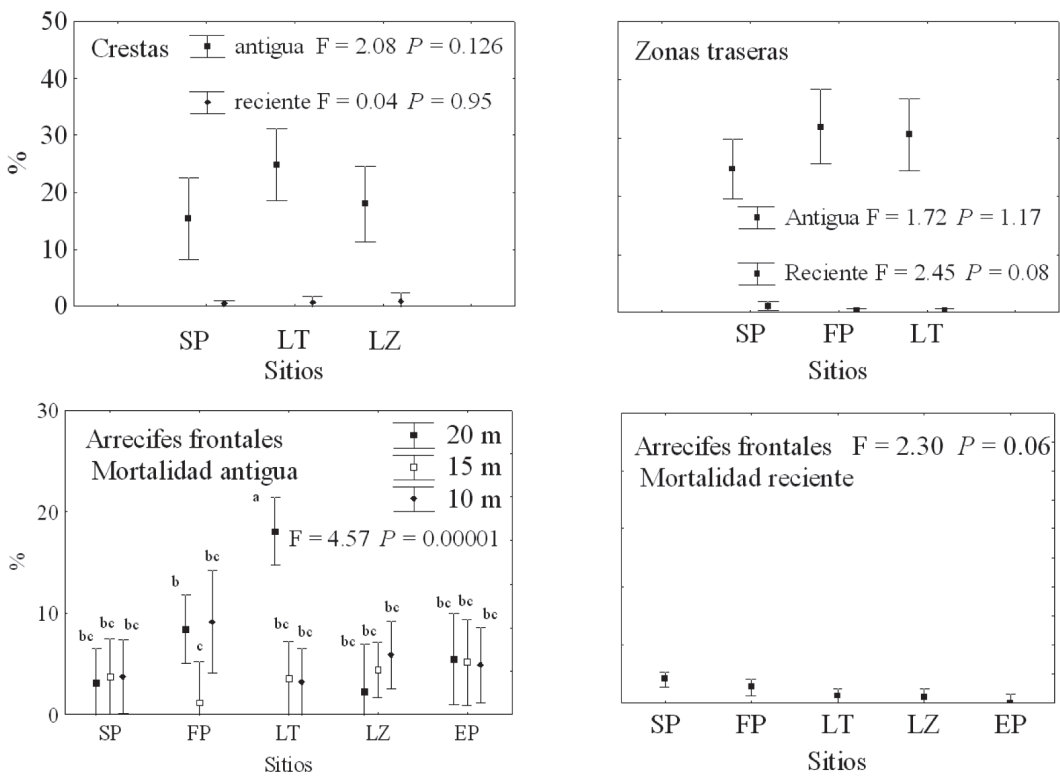

Fig. 5. Mortalidades antigua y reciente (media e intervalos de confianza) de los corales en las zonas ecológicas del arrecife. Las letras muestran la significación de las diferencias. Ver siglas de los sitios en la Fig. 2

Fig 5. Old and recent coral mortalities (mean and confidence intervals) of the reef ecological zones. Letters indicate significance of differences. Site codes as in Fig. 2 
analizadas. En las crestas de Sancho Pardo y La Tabla predominó A. palmata, mientras que en La Zorrita lo hizo P. astreoides. Se destacaron, además, por su abundancia Millepora complanata (Linnaeus, 1758) en todos los sitios, y Diploria strigosa (Ellis y Solander, 1786) y D. clivosa (Ellis y Solander, 1786) en La Tabla (Cuadro 1). En las zonas traseras dominó marcadamente M. annularis. En Sancho Pardo también resaltó por su cubrimiento A. cervicornis (Cuadro 1).

De forma general, en los arrecifes frontales, la especie con mayor cubrimiento fue $S$. siderea. Sin embargo, se apreció variabilidad en el cubrimiento vivo del sustrato entre localidades y profundidades por un grupo de especies. $M$. faveolata (Ellis y Solander, 1786) dominó en los 15 y 20 m de Sancho Pardo, y en los $20 \mathrm{~m}$ de Francisco Padre y La Tabla. Sobresale la abundancia de $P$. porites (Pallas, 1766) en los $10 \mathrm{~m}$ de Francisco Padre y La Tabla, los $15 \mathrm{~m}$ de Francisco Padre y El Pinto, igual que en los $20 \mathrm{~m}$ de este último. En El Pinto resalta la abundancia de $M$. cavernosa, a diferencia del resto de los sitios, igual que $S$. intersepta en los 20 $\mathrm{m}$ de Sancho Pardo y Agaricia agaricites (Linnaeus, 1758) en los $15 \mathrm{~m}$ de La Zorrita (Cuadro 1).

Los porcentajes de cubrimiento por especies tolerantes a los sedimentos (\% ETS) fueron mayores en la cresta de La Zorrita que en las restantes estaciones de cresta y en esto influyeron los cubrimientos de P. astreoides y Diploria spp. En las zonas traseras fueron muy parecidos entre sitios e inferiores a los de la cresta. En los arrecifes frontales, los \% ETS mostraron la tendencia de incrementarse en la profundidad de $10 \mathrm{~m}$, a excepción de El Pinto, cuyas tres profundidades presentaron valores por encima de $80 \%$ (en esto influyeron $S$. siderea, M. cavernosa y $P$. porites), los mayores registros para el arrecife (Cuadro 1).

Los valores de cubrimiento de $P$. astreoides no fueron bajos, a pesar de la poca talla de las colonias y responden a la alta densidad y baja mortalidad de esta especie. M. annularis sobresalió por su cubrimiento en las zonas traseras, aunque igualmente sus porcentajes de mortalidad no son bajos, igual que $A$. cervicornis, la especie con más daños parciales y totales (Fig. 6). M. faveolata presentó mayor cubrimiento, talla y menor mortalidad en los arrecifes frontales de Sancho Pardo y Francisco Padre. En La Tabla mostró la mayor mortalidad, mientras que en E1 Pinto prácticamente no estuvo presente. $\mathrm{La}$ situación de $S$. siderea parece ser similar entre sitios con respecto a daños, aunque tendió a incrementar sus porcentajes y tallas hacia el oeste del arrecife. P. porites prácticamente no estuvo presente en Sancho Pardo, sin embargo, su cubrimiento, talla y ausencia de daños fueron mayores en Francisco Padre. En este mismo sitio, A. agaricites estuvo casi ausente y esta especie mostró un ligero comportamiento de deterioro de su condición hacia el este del arrecife (Fig. 6).

\section{DISCUSIÓN}

La condición general de los corales de las crestas fue de deterioro. En Francisco Padre y en El Pinto se observó una reducción del relieve arrecifal ("aplanamiento", denominación de Alvarez-Filip et al. 2009) y, por lo tanto, una disminución de disponibilidad de refugio para la biota. En Francisco Padre, la desaparición de colonias vivas de $A$. palmata (en este sitio se encontraron evidencias de poblaciones anteriormente vivas de esta especie, no así 


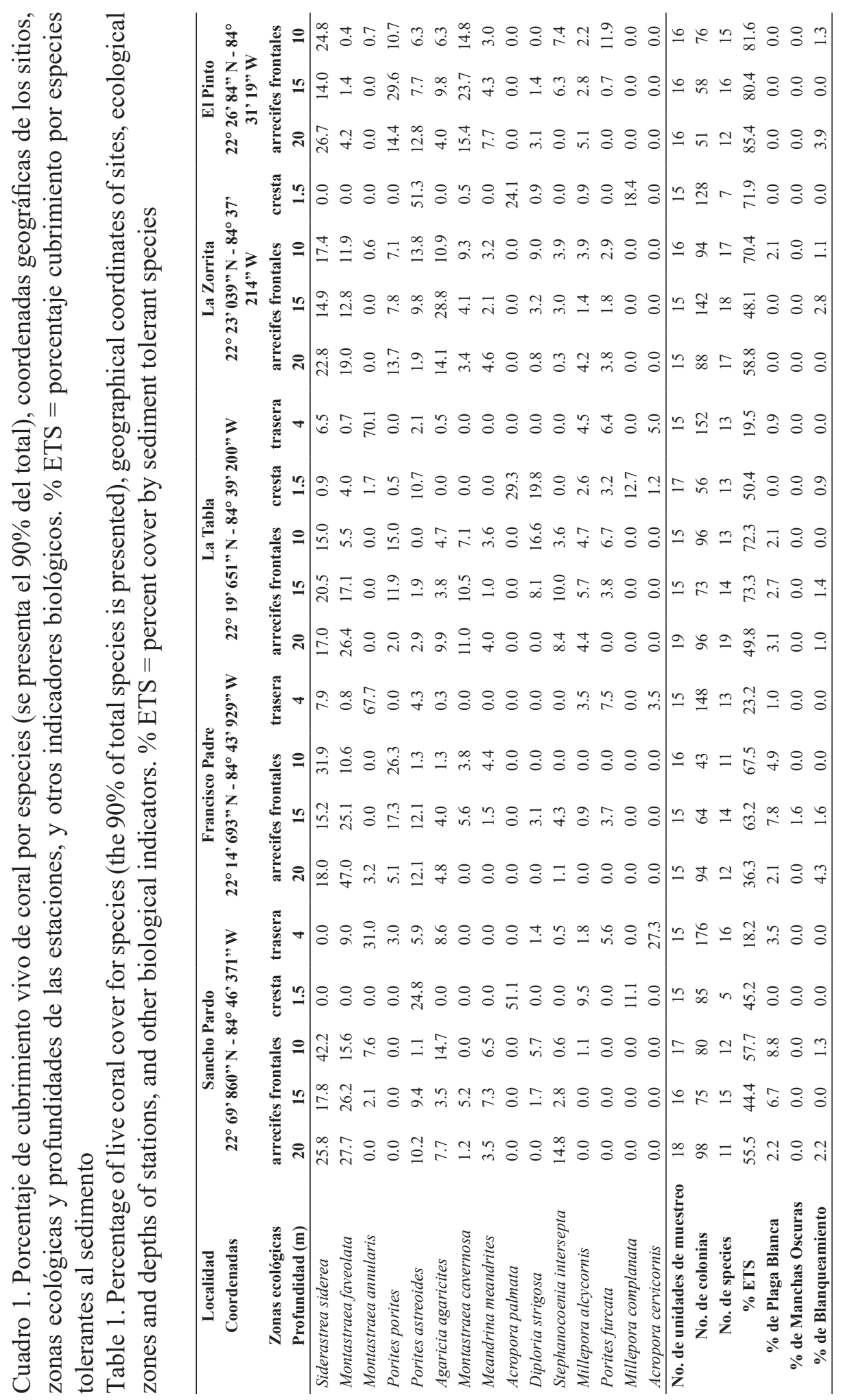



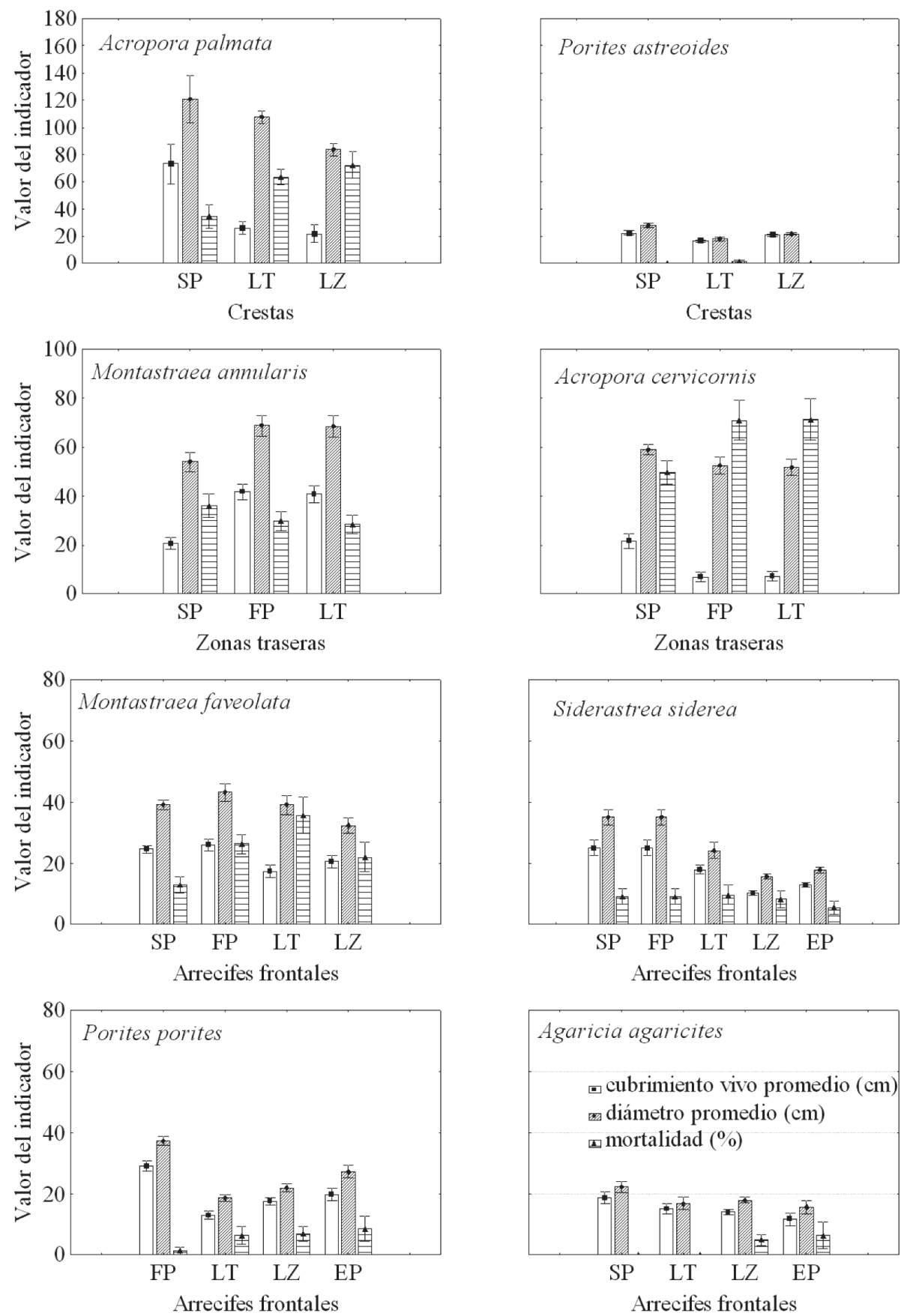

Fig. 6. Cubrimiento, talla y mortalidad en las especies más abundantes. Ver siglas de los sitios en la Fig. 2

Fig. 6. Cover, size y mortality of the most abundant species. See codes of sites in Fig. 2 
en El Pinto, donde no hay información al respecto ni se vieron restos de colonias in situ) y la escasez de reclutas o indicios de recubrimiento con tejido vivo vislumbran una recuperación difícilmente probable de esta especie, si las condiciones ambientales causantes se mantienen.

En las tres crestas restantes, el cubrimiento vivo de coral está por encima de la media actual para Cuba (17.6\%, según revisión de los años 2003 a 2009 por Alcolado et al. 2009a) y se acerca a la media reportada por Kramer (2003) en sus observaciones sobre registros en el Gran Caribe (25\%), conforme a AGRRA 1999-2001. Sin embargo, a excepción del sitio Sancho Pardo, A. palmata no aporta de forma significativa este indicador. Comparten predominio con ella Diploria spp. y $P$. astreoides; esta última especie se considera un estratega $r$ muy resistente a disturbios tensodinámicos y de sedimentación (Torres y Morelock, 2002). En la actualidad se ha observado que ha incrementado notablemente su dominio en crestas afectadas por eventos extremos, gracias a su resistencia y poder reproductivo. Sin embargo, como tal no contribuye marcadamente al relieve de estas, por lo que no es apta como refugio por su hábito incrustante (Alcolado et al. 2009b).

Los promedios de cubrimiento vivo de coral de las zonas traseras son comparativamente menores a los registrados por Caballero et al. $(2004,2009)$ en Bahía de Cochinos (sur central de Cuba) y el litoral La Habana (nordeste de Cuba), sitios de fondos parecidos de cabezos de coral someros y con similar composición por especies, pero históricamente menos impactados por el paso de huracanes (Lewis y Hsu, 1992). Se asemejan a sitios de arrecifes de parche de los cayos de La Florida, que han sido impactados igualmente por huracanes y eventos de blanqueamiento y han sufrido una disminución de la cobertura viva de coral de un 20 a un $15 \%$ en el transcurso de diez años (Causey, 2008).

La mortalidad parcial en $M$. annularis y A. cervicornis (especies dominantes) influyó decisivamente en que no se alcanzasen mayores porcentajes de cubrimiento vivo de coral en las zonas traseras. No sería errado atribuir al paso de los huracanes los daños parciales y totales que muestran ambas especies en los sitios menos profundos, sumado al efecto de los frentes fríos, eventos que actúan por mediación, tanto del impacto mecánico como del efecto de los sedimentos, ambos generados por el fuerte oleaje. McField (2000) reportó una reducción drástica en la cobertura coralina (entre 36 y $62 \%$ ) en segmentos de arrecifes someros de Belice, siendo la especie más afectada $A$. cervicornis. Un huracán puede contribuir a incrementar el cubrimiento del género Acropora, gracias a su capacidad de fragmentación y supervivencia de sus fragmentos. Sin embargo, una mayor frecuencia de huracanes puede reducir considerablemente la cobertura de este (Lirman y Fong, 1997). Se coincide en considerar a A. cervicornis como una especie sensible al oleaje (Geister, 1977) y sus poblaciones vienen siendo las más afectadas en el Caribe ante los disturbios naturales de finales del siglo XX y principios del siglo XXI (Alcolado et al. 2001; Aronson y Precht, 2001; Caballero et al. 2004, 2009; Acropo$r a$ Biological Review Team, 2005).

Los valores medios de cubrimiento vivo en la mayor parte de los sitios de arrecifes frontales también se encuentran por debajo de la media actual reportada para Cuba (13.4\%, según revisión de los años 2003 a 2009 por Alcolado et al. 2009a) y para el Atlántico Occidental a inicios de siglo (26\%, Kramer, 2003). Estos registros, 
igual que los de densidad y tallas, son parecidos a los reportados por Busutil et al. (en preparación) en arrecifes frente a Playa Santa Lucía, noroeste de Cuba, que se encuentran bajo un constante estrés natural, a causa de la acción combinada del oleaje generado por exposición abierta y crónica a los vientos alisios directamente del Océano Atlántico, junto con la resuspensión natural del sedimento del fondo, que provoca deposición y abrasión sobre los corales.

En los arrecifes frontales, sobre todo entre los 10 y 15 m, se evidenció una condición ligeramente mejor en los sitios ubicados hacia el oeste (mayor cubrimiento, talla de los corales y predominio de $M$. faveolata), zona donde los vientos alisios inciden de forma más tangencial. Lo contrario ocurre hacia el este, donde los vientos inciden de manera más frontal. En esos sitios se observó una marcada tendencia al incremento de las especies más tolerantes al efecto de los sedimentos. En los 20 $\mathrm{m}$ de profundidad, este patrón fue menos evidente, al parecer debido a que generalmente a esta profundidad los efectos físicos del oleaje disminuyen su acción sobre los corales e influyen, de forma más activa, los efectos biológicos estocásticos.

En los arrecifes frontales, la mortalidad antigua encontrada fue baja y podría responder a cualquier causa natural (como una mayor oportunidad y consecuente rapidez para la recuperación de la cobertura coralina), y no tanto específicamente a la acción física de los huracanes, dada la mayor profundidad. En el sitio Sancho Pardo se encontró mucho sustrato coralino muerto (en los restantes predominó como sustrato un pavimento rocoso), pero con alto grado de erosión que imposibilitó la identificación de colonias de coral, por lo que este se interpretó como sustrato y no como coral muerto, $y$ su porcentaje no se incluyó en el porcentaje de mortalidad antigua. Esto puede reflejar que en un pasado lejano, este sitio tuvo mayor cubrimiento vivo de coral y concuerda con el análisis del párrafo anterior, donde se plantean las ventajas de la posición más resguardada de este sitio ante los vientos del este y noroeste $\mathrm{y}$, por lo tanto, menor disturbio hidrodinámico sobre procesos como el reclutamiento en general y el establecimiento y crecimiento de especies más sensibles a los efectos de los sedimentos.

Según Heron et al. (2008), los corales responden de forma adaptativa al paso de un huracán con un incremento de su tasa de reclutamiento. Posiblemente este proceso adaptativo en los arrecifes frontales de Sancho Pardo se vea bastante afectado por la disponibilidad de sustrato para el asentamiento de los reclutas, debido a la abundancia de algas que lo cubren. Esto parece ser causado por la carencia de herbívoros, en especial, los erizos negros de espinas largas, $D$. antillarum. Otro factor que debe restringir en cierta medida el reclutamiento en esta zona es la abierta exposición de estos arrecifes a los fuertes vientos de los frentes fríos durante la temporada invernal, pero este es un efecto que históricamente debe haber sido parte habitual de la dinámica a largo plazo de los arrecifes en el área de estudio.

Los porcentajes de enfermedades en los arrecifes frontales de los sitios Sancho Pardo y Francisco Padre (8.8\% y 7.4\%, respectivamente) fueron superiores a los reportados por De la Guardia et al. (2005) en Cayo Levisa $3.5 \%$ de colonias afectadas), Bahía de Cochinos (3\%, Caballero et al. 2007) y Archipiélago de los Canarreos (menos de 1\%, Alcolado et al. 2010), y parecidos a los de cerca de río Mosquito, al norte de La Habana (6\%, Caballero et al. 2009). No se descarta que las mortalidades parciales encontradas en M. faveolata 
fueran causas de moderados pero frecuentes brotes de enfermedades en el área. La plaga blanca se ha visto afectando fundamentalmente a esta especie en arrecifes de Guanahacabibes, donde se ha observado una disminución de cubrimiento vivo de esta (Caballero et al. 2007; Perera, 2010).

\section{CONCLUSIONES}

- La condición observada de los arrecifes estudiados parece ser consecuencia de diferentes combinaciones e intensidades de factores que varían en espacio y tiempo, que incluyen: una mayor frecuencia reciente de huracanes, la recurrente exposición a los frentes fríos, enfermedades de corales (posiblemente incluido el blanqueamiento), abundancia de macroalgas favorecida por un bajo nivel de herbivorismo, y efecto de la profundidad y del grado de exposición a los vientos.

- La combinación de los factores señalados podría limitar la resiliencia de los arrecifes simultáneamente por daño directo a los corales establecidos, y por restricción de la recuperación de las colonias perjudicadas y del reclutamiento.

- La condición de los corales en las crestas de los Bajos de Sancho Pardo fue de deterioro, ya que la cobertura del fondo por A. palmata en general es baja, y esta especie se encuentra ausente o totalmente muerta en algunos sitios, sin indicios de suficiente reclutamiento ni recubrimiento por tejido vivo.

- La condición de los corales en las zonas traseras fue, de manera comparativa, moderadamente favorable, a pesar de los porcentajes relativamente altos de mortalidad de $M$. annularis y A. cervicornis como posible consecuencia del reciente aumento de la frecuencia de huracanes y la incidencia de enfermedades de corales.
- Los arrecifes frontales evidenciaron estar sometidos a un estrés moderado pero crónico, al parecer en parte provocado por los vientos del norte y noroeste de los frentes fríos, que impiden que se alcancen mayores porcentajes de cubrimiento. Por otra parte, las enfermedades y el elevado cubrimiento del sustrato por algas carnosas deben estar contribuyendo a la disminución del cubrimiento con escasas posibilidades de recuperación mediante reclutamiento.

\section{BIBLIOGRAFÍA}

Acevedo, R., Morelock, J. \& Olivieri, R. A. (1989). Modification of Coral Reef Zonation by Terrigenous Sediment Stress. PALAIOS, 4, 92-100.

Acropora Biological Review Team. (2005). Atlantic Acropora Status Review Document. Report to National Marine Fisheries Service, Southeast Regional Office. Alcolado, P. M. (1981). Zonación de los gorgonáceos someros de Cuba y su posible uso como indicadores comparativos de tensión hidrodinámica sobre los organismos del bentos. Inf. Cient. Téc. Inst. Oceanol., 187, 1-43.

Alcolado, P. M. (2010). El cambio climático y los arrecifes coralinos del Gran Caribe y Cuba. En A. Hernández-Zanuy \& P. M. Alcolado (Eds.), La biodiversidad en ecosistemas marinos y costeros del litoral de iberoamérica y el cambio climático: I. Memorias del Primer Taller de la Red CYTED BIODIVMAR, La Habana, Cuba. (CD-ROM). La Habana, Cuba: Instituto de Oceanología.

Alcolado, P. M., Claro-Madruga, R., Martínez-Daranas, B., Menéndez-Macía, G., García-Parrado, P., Cantelar, K., Hernández, M. \& del Valle, R. (2001). Evaluación ecológica de los arrecifes 
coralinos del oeste de Cayo Largo del Sur, Cuba: 1998-1999. Bol. Invest. Mar. Cost., 30, 109-132.

Alcolado, P. M., Caballero, H. \& Perera, S. (2009a). Tendencia del cambio en el cubrimiento vivo por corales pétreos en los arrecifes coralinos de Cuba. Serie Oceanológica, 5, 1-14.

Alcolado, P. M., Hernández-Muñoz, D., Caballero, H., Busutil, L., Perera, S. \& Hidalgo, G. (2009b). Efectos de un inusual período de alta frecuencia de huracanes sobre el bentos de arrecifes coralinos. Rev. Mar. y Cost., 1, 73-93.

Alcolado, P. M., Morgan, I. E., Kramer, P. A., Ginsburg, R. N., Blanchon, P., De la Guardia, E., Kosminin, V., GonzálezFerrer, S. \& Hernández, M. (2010). Condición de arrecifes remotos en el suroeste de Cuba. Cienc. Mar., 36(2), 179-197.

Alvarez-Filip, L., Dulvy, N. K., Gill, J. A., Côté, I. M. \& Watkinson, A. R. (2009). Flattening of Caribbean coral reefs: region-wide declines in architectural complexity. Proc. R. Soc. B., 276, 3019-3025.

Aronson, R. B. \& Precht, W. F. (2001). White-band disease and the changing face of Caribbean coral reefs. Hydrobiologia, 460, 25-38.

Caballero, H., Varona, G. \& García, Y. (2004). Estructura ecológica de las comunidades de corales de Bahía de Cochinos, Cuba. Rev. Invest. Mar, 25(1), 23-26.

Caballero, H., González-Ferrer, S., Cobian, D., Álvarez, S. \& Alcolado-Prieto, P. (2007). Evaluación AGRRA del bentos de diez sitios de buceo de María La Gorda, Bahía de Corrientes, Cuba. Rev. Invest. Mar., 28(2), 131-138.

Caballero, H., Alcolado, P. M. \& Semidey, A. (2009). Condición de los arrecifes de coral frente a costas con asentamientos humanos y aportes terrígenos:
El caso del litoral habanero, Cuba. Rev. Mar. y Cost., 1, 49-72.

Causey, B. D. (2008). Coral Reefs of the U.S. Caribbean. The history of massive coral bleaching and other perturbation in the Florida Keys. En C. Wilkinson \& D. Souter (Eds.), Status of Caribbean Coral Reefs after Bleaching and Hurricanes in 2005 (pp. 61-67). Townsville, Australia: Global Coral Reef Monitoring Network, and Reef and Rainforest Research Centre.

De la Guardia, E., González-Díaz, P., Valdivia, A. \& González-Sansón, G. (2005). Características generales de los arrecifes coralinos en la zona de buceo de Cayo Levisa, Archipiélago de los Colorados, Cuba. Rev. Invest. Mar., 26(1), 37-44.

Geister, J. (1977). The influence of wave exposure on the ecological zonation of Caribbean coral reefs. Proc. 3rd Int. Coral Reef Symp., Miami, Fl., 2, 23-29. González-Díaz, P., González-Sansón, G., Álvarez-Fernández, S. \& Perera-Pérez, O. (2010). High spatial variability of coral, sponges and gorgonian assemblages in a well preserved reef. Rev. Biol. Trop., 58(2), 621-634.

González-Ferrer, S. (2009). Diversidad de Organismos. Celenterados-Filo CNIDARIA: Clase ANTHOZOA, Subclase ZOANTHARIA, corales pétreosOrden SCLERACTINIA. En R. Claro (Ed.), Biodiversidad marina de Cuba (pp. 42-46). La Habana, Cuba: Instituto de Oceanología.

González-Sansón, G., Aguilar, C., Hernández, I., Cabrera, Y. \& Curry, A. (2009). The influence of habitat and fishing on reef fish assemblages in Cuba. Gulf Carib. Res., 21, 13-21.

Heron, S., Morgan, J., Eakin, M. \& Skirving, W. (2008). Hurricanes and their 
effects on coral reefs. En C. Wilkinson \& D. Souter (Eds.), Status of Caribbean Coral Reefs after Bleaching and Hurricanes in 2005 (pp. 31-36). Townsville, Australia: Global Coral Reef Monitoring Network, and Reef and Rainforest Research Centre.

Hoegh-Guldberg, O., Mumby, P. J., Hooten, A. J., Steneck, R. S., Greenfield, P., Gomez, E., Harvell, C. D., Sale, P. F., Edwards, A. J., Caldeira, K., Knowlton, N., Eakin, C. M., Iglesias-Prieto, R., Muthinga, N., Bradbury, R. H., Dubi, A. \& Hatziolos, M. E. (2007). Coral reefs under rapid climate change and ocean acidification. Science, 318(5857), 1737-1742.

Kramer, P. (2003). Synthesis of coral reef health indicators for the western Atlantic: Result of the AGRRA program (1997-2000). Atoll Res. Bull., 496, 1-57.

Kramer, P. \& Lang, J. (2003). The Atlantic and Gulf Rapid Reef Assesment (AGRRA) protocols: former version 2.2. Atoll Res. Bull., 496(35), 611-624.

Lewis, J. K. \& Hsu, S. A. (1992). Mesoscale air-sea interactions related to tropical and extratropical storms in the Gulf of Mexico. J. Geophys. Res., 97(C2), 2215-2228.

Lirman, D. \& Fong, P. (1997). Susceptibility of coral communities to storm intensity, duration and frequency. Proc. 8th Int. Coral Reef Symp., Panamá, 1, 561-566.

Lluis-Riera, M. (1983). Estudios hidrológicos de la plataforma noroccidental de Cuba (zona c). Rep. Invest. Inst. Oceanología, 13, 1-33.

McField, M. D. (2000). Influence of disturbance on coral reef community structure in Belice. Proc. 9th Int. Coral Reef Sym., Bali, Indonesia, 1, 23-27.

Obura, D. O. \& Grimsditch, G. (2008). Resilience Assessment of Coral
Reefs-Assessment protocol for coral reefs, focusing on coral bleaching and thermal stress. Gland, Switzerland: IUCN Working Group on Climate Change and Coral Reefs.

Perera, S. (2010). Influencia de los vientos generadores de oleaje sobre comunidades de corales en el Parque Nacional Guanahacabibes, Cuba. Tesis para el grado de Máster en Ecología Marina no publicada. Universidad de La Habana, Cuba.

Richardson, L. L. (1998). Coral diseases, what is really known? Trends Ecol. Evol., 13(11), 438-443.

Rogers, C. S. (1990). Responses of coral reefs and reef organisms to sedimentation. Mar. Ecol. Prog. Ser., 62, 185-202.

Torres, J. \& Morelock, J. (2002). Effect of terrigenous sediment influx on coral cover and linear extension rates of three Caribbean massive coral species. Caribb. J. Sci., 38(3-4), 222-229.

Veron, J. E. N., Hoegh-Guldberg, O., Lenton, T. M., Lough, J. M., Obura, D. O., Pearce-Kelly, P., Sheppard, C. R. C., Spalding, M., Stafford-Smith, M. G. \& Rogers, A. D. (2009). The coral reef crisis: The critical importance of $<350 \mathrm{ppm}$ $\mathrm{CO}_{2}$. Mar. Poll. Bull., 58, 1428-1436.

Walton-Smith, F. G. (1948). The Atlantic Reef Corals. Coral Gables, Florida, EE.UU.: University of Miami Press.

Wilkinson, C. R. (2000). Status of Coral Reefs in the World: 2000. Townsville, Australia: Institute of Marine Science. Wilkinson, C. \& Souter, D. (2008). Status of Caribbean Coral Reefs After Bleaching and Hurricanes in 2005. Townsville, Australia: Global Coral Reef Monitoring Network, and Reef and Rainforest Research Centre.

Zar, J. H. (1996). Biostatistical Analysis. New Jersey, EE.UU.: Prentice Hall. 